\title{
ANALYSIS OF MOBILE TELECOMMUNICATION PATH LOSS IN RURAL COMMUNITIES
}

\author{
ALEXANDER OKANDEJI ${ }^{1 *}$, FRANK ONAIFO ${ }^{2}$, AYODEJI OKUBANJO ${ }^{2}$, \\ MATHEW OLAJIDE ${ }^{2}$, HEZEKIAH FASANYA ${ }^{3}$ \\ ${ }^{I}$ Department of Electrical/Electronic Engineering, University of Lagos, Akoka, Lagos, \\ Nigeria \\ ${ }^{2}$ Dept. of Elect/Elect Engineering, Olabisi Onabanjo University, Ogun State, Nigeria. \\ ${ }^{3}$ Dept. of Computer Engineering, Olabisi Onabanjo University, Ogun State, Nigeria.
}

\begin{abstract}
In this work, a cheaper alternative method of determining path loss using Network cell info lite software is proposed. Hata-Okumura model is used in the determination of path loss and signal strength of mobile communication devices within Ibogun and Ifo, a suburban community in Ogun state, Nigeria. Additionally, this paper is also aimed at determining the path loss under transmission line to ensure proper network planning in areas covered by transmission lines. Result obtained shows that areas with pylons have greater path loss compared to areas with no pylons. It is recommended that the power levels of base station operating in this area should be increased.
\end{abstract}

Keywords: Hata-Okumura, path loss, signal strength, network planning, quality of service

\section{INTRODUCTION}

Path Loss is the decrease in the power density of an electromagnetic wave between the transmitter and the receiver [1]. Path loss models describe the signal weakening between a transmitting and a receive antenna as a function of the propagation distance and other parameters. Some models include many details of the terrain profile to estimate the signal attenuation, whereas others just consider carrier frequency and distance. The heights of antennas of both base station and mobile receiver are also critical parameters when modelling path loss. In general, the losses present in a signal during propagation from base station to receiver may be classical and already exiting. General classification includes three forms of modelling namely: empirical, semi deterministic, and deterministic models, to analyse these losses [2].

The empirical model comprises the use of fast calculations to achieve path loss modelling but have accuracy problems in different areas. The semi deterministic model on the other hand such as Walfisch-Ikegami (W-I) model requires a ground survey of the environment to provide high accuracy prediction. In contrast, the deterministic model provides a very high accuracy in path loss prediction but require extensive detail of the environment; thus, they require a higher calculation time [3].

Authors in [3] investigated the comparison between mixing and pure W-I path loss models for cellular mobile communication network. Accordingly, work done in [3] proposed a method of classifying objects on 2D aerial

\footnotetext{
${ }^{*}$ Corresponding author email: aokandeji@unilag.edu.ng

(C) 2020 Alma Mater Publishing House
} 
image by using maximum likelihood algorithm (MLA) to identify objects in the area, divided them into grids, and then classify them into different categories i.e. road, plain, building, forest and water in order to determine the type of area of propagation into free space, rural, forest, suburban, urban and dense-urban areas. This was done using fuzzy logic with respect to inputs from the grid categories.

In like manner, authors in [4] investigated the effect of Clutters on Path Loss proposed by W-I Propagation Model. Work done in [4] proposed a modified model of the W-I model by adding the effect of vegetation, sea, snowfall and rain. Accordingly, simulation result was used to illustrate the effect of disorganized obstructions on path loss proposed by W-I propagation model.

Several models have been developed to determine path loss but none of these models have been able to address all the challenges of accurately modelling all the loss component of an electromagnetic signal. Example of these models include: Egli model which was derived from real-world data on ultra-high frequency (UHF) and very high frequency (VHF) television transmissions in several large cities, and it predicts the path loss for a point-to-point link. The Hata-Okumura model on the other hand was built using data collected in the city. Accordingly, this model is ideal for use in cities with many urban structures. Authors in [5] modelled signal propagation considering highly obstructed and less obstructed areas using a mobile signal analyzer. Result obtained in [5] showed that the network that uses higher effective isotropic radiated power (EIRP) value of $64.5 \mathrm{dBm}$ with lower antenna gain of $17.5 \mathrm{~dB}$ covered more distance up to $3000 \mathrm{~m}$ from the $\mathrm{BS}$ transmitter before fading below $-100 \mathrm{~dB}$ compared to the network that uses lower EIRP of $64.00 \mathrm{dBm}$ and higher gain of $18 \mathrm{~dB}$, which faded faster below $-100 \mathrm{dBm}$ from distance of $2250 \mathrm{~m}$.

Authors in [6] investigated the optimized path loss model for the effects of environmental factors on mobile signal strength. Result obtained in [6] show that incorporating environmental parameters has the potential to give accurate path loss predictions. Other models include: Okumura-Hata model which is used to predict the path loss of cellular transmissions in exterior environments [7, 8]. Lee Model which is a relatively simple, intuitive model which provides reasonably accurate path loss predictions, and Walfisch- Ikegami model which facilitates radio frequency path loss predictions in typical sub-urban and urban environments [7].

In contrast to existing results, this work proposes a cheaper alternative method of determining path loss using Network-cell-info-lite software. In particular, Hata-Okumura model is used in the determination of path-loss and signal strength of mobile communication devices within Ibogun and Ifo Community (a rural community in Ogun state, Nigeria - Figure 1). Additionally, this paper considers the determination of path loss under transmission line to ensure proper network planning in areas covered by transmission lines. This is because several communities in Lagos and other parts of Nigeria are criss-crossed by Pylon (transmission line).

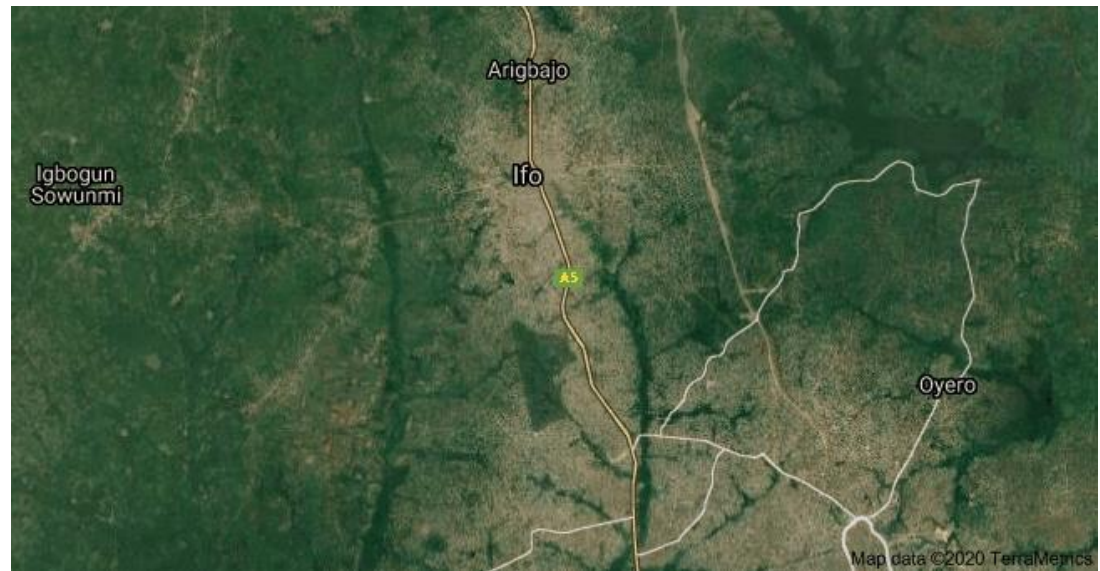

Fig. 1. Satellite Map of IFO, Ogun State, Nigeria.

\section{EXPERIMENTAL SET UP}

The area covered in this work is the Olabisi Onabanjo University, Ibogun Campus, Ibogun, Ogun state, Nigeria. We investigate the received mobile signal measurements and data collection at the cellular mobile fields of the two mobile service networks available in Ibogun community. The received signal power density is measured and 
collected with the use of a cellular mobile network analyser named Network-cell-info-lite which is capable of measuring signal power density in decibel milli watts $(\mathrm{dBm})$.

Some selected buildings such as the Electrical, Computer, Mechanical, Civil engineering buildings as well as selected houses within Ibogun campus were considered while taking the drive test.

\subsection{Experimental Measurements}

Experimental measurements were obtained at designated locations with the use of a mobile application (NetworkCell-Info-Lite) capable of measuring received signal power in decibel milliwatts $(\mathrm{dBm})$. During measurements, readings of received signal powers were taken as the motorcycle moved away from the serving base stations. The first reading was carried out at Ifo road to Ibogun with the mobile software, and measurements such as the received signal, height, longitude, latitude and some other relevant values were recorded. The Hata-Okumura model includes adjustments to the basic equation to account for Urban, Suburban and Open area propagation losses (eq. $1-4)$.

$$
\begin{aligned}
& A+B \log 10(d) ; \text { urban area } \\
& \text { Lp }=A+B \log 10(d)-C ; \text { suburban area } \\
& A+B \log 10(d)-D ; \text { open area, }
\end{aligned}
$$

where,

$\mathrm{A}=69.55+26.16 \log 10\left(\mathrm{f}_{\mathrm{c}}\right)-13.82 \log 10\left(\mathrm{~h}_{\mathrm{b}}\right)-\mathrm{a}\left(\mathrm{h}_{\mathrm{m}}\right) ; \mathrm{B}=44.9-6.55 \log 10\left(\mathrm{~h}_{\mathrm{b}}\right) ; \mathrm{C}=5.4+2\left[\log 10\left(\mathrm{f}_{\mathrm{c}} / 28\right)\right] ; \mathrm{D}=$ $40.94+4.78\left[\log 10\left(\mathrm{f}_{\mathrm{c}}\right)\right]-18.33 \log 10\left(\mathrm{f}_{\mathrm{c}}\right) ;$ and $\mathrm{a}\left(\mathrm{h}_{\mathrm{m}}\right)=\left[1.1 \log 10\left(\mathrm{f}_{\mathrm{c}}\right)-0.7\right] \mathrm{h}_{\mathrm{m}}-1.56 \log 10\left(\mathrm{f}_{\mathrm{c}}\right)-0.8 ;[$ medium cities].

$$
\mathrm{L}=69.55+26.16 \log \mathrm{f}_{\mathrm{c}}-13.82 \log \mathrm{h}_{\mathrm{b}}-\mathrm{a}\left(\mathrm{h}_{\mathrm{m}}\right)+\left(44.9-6.55 \log \mathrm{h}_{\mathrm{b}}\right) \log \mathrm{d}(\mathrm{dB}) .
$$

where, $\mathrm{f}_{\mathrm{c}}=$ frequency in MHz; $\mathrm{L}=$ mean path loss in $\mathrm{dB} ; \mathrm{h}_{\mathrm{b}}=\mathrm{BS}$ antenna height in $\mathrm{m} ; \mathrm{a}\left(\mathrm{h}_{\mathrm{m}}\right)=$ correction factor for mobile antenna height in $\mathrm{dB}$; $\mathrm{d}=$ distance from base station in $\mathrm{Km}$.

\section{RESULTS AND DISCUSSIONS}

\subsection{Results}

The values of simulated path-loss/experimental path-loss atc1920-1955 MHz/1835-1850 MHz in a sub-urban area are given in the Tables $1-2$.

Table 1. Simulated path-loss at $1920-1955 \mathrm{MHz}$ in a sub-urban area.

\begin{tabular}{|c|c|c|c|c|}
\hline \multirow{2}{*}{ S/N } & \multirow{2}{*}{ Distance $(\mathbf{m})$} & \multirow{2}{*}{ Signal $(\mathbf{d B})$} & \multicolumn{2}{|c|}{ L (dB) } \\
\cline { 3 - 5 } & & & Simulated & Experimental \\
\hline 1 & 200 & -85 & 110.5669 & 146.115 \\
\hline 2 & 400 & -91 & 121.3268 & 152.115 \\
\hline 3 & 600 & -91 & 127.6209 & 152.115 \\
\hline 4 & 800 & -85 & 132.0866 & 146.115 \\
\hline 5 & 1000 & -97 & 135.5505 & 158.115 \\
\hline 6 & 1200 & -97 & 138.3808 & 158.115 \\
\hline 7 & 1400 & -97 & 140.7737 & 158.115 \\
\hline 8 & 1600 & -97 & 142.8465 & 152.115 \\
\hline 9 & 1800 & -91 & 144.6749 & 146.115 \\
\hline 10 & 2000 & -85 & 146.3104 & 146.115 \\
\hline 11 & 2200 & -85 & 147.7899 & 152.115 \\
\hline 12 & 2400 & -91 & 149.1406 & 146.115 \\
\hline 13 & 2600 & -85 & 150.3831 & 146.115 \\
\hline 14 & 2800 & -85 & 151.5335 & 146.115 \\
\hline 15 & 3000 & -85 & 152.6045 & \\
\hline
\end{tabular}




\begin{tabular}{|l|l|l|l|l|}
\hline 16 & 3200 & -85 & 153.6064 & 146.115 \\
\hline 17 & 3400 & -91 & 154.5475 & 152.115 \\
\hline
\end{tabular}

Table 2. Simulated/experimental path-loss at $1835-1850 \mathrm{MHz}$ in a sub-urban area.

\begin{tabular}{|c|c|c|c|c|}
\hline \multirow{2}{*}{ S/N } & \multirow{2}{*}{ Distance (m) } & \multirow{2}{*}{ Signal (dB) } & \multicolumn{2}{|c|}{$\mathbf{L}(\mathbf{d B})$} \\
\cline { 3 - 5 } & & & Simulated & Experimental \\
\hline 1 & 200 & -77 & 109.268 & 138.115 \\
\hline 2 & 400 & -77 & 119.872 & 138.115 \\
\hline 3 & 600 & -79 & 126.0747 & 140.115 \\
\hline 4 & 800 & -79 & 130.4757 & 140.115 \\
\hline 5 & 1000 & -81 & 133.8893 & 142.115 \\
\hline 6 & 1200 & -81 & 136.6785 & 142.115 \\
\hline 7 & 1400 & -79 & 139.0367 & 140.115 \\
\hline 8 & 1600 & -91 & 141.0794 & 152.115 \\
\hline 9 & 1800 & -81 & 142.8813 & 142.115 \\
\hline 10 & 2000 & -83 & 144.4931 & 144.115 \\
\hline 11 & 2200 & -83 & 145.9511 & 144.115 \\
\hline 12 & 2400 & -97 & 147.2822 & 158.115 \\
\hline 13 & 2600 & -97 & 148.5067 & 158.115 \\
\hline 14 & 2800 & -95 & 149.6404 & 156.115 \\
\hline 15 & 3000 & -95 & 150.6959 & 156.115 \\
\hline 16 & 3200 & -95 & 151.6832 & 156.115 \\
\hline 17 & 3400 & -101 & 152.6106 & 162.115 \\
\hline
\end{tabular}

The simulated/experimental path-loss under transmission line are given in the Table 3.

Table 3. Simulated/experimental path-loss under transmission line.

\begin{tabular}{|c|c|c|c|c|}
\hline \multirow{2}{*}{ S/N } & \multirow{2}{*}{ Distance(m) } & \multirow{2}{*}{ Signal(dB) } & \multicolumn{2}{|c|}{ L(dB) } \\
\cline { 3 - 5 } & & & Simulated & Experimental \\
\hline 1 & 200 & -115 & 109.268 & 176.115 \\
\hline 2 & 400 & -107 & 119.872 & 168.115 \\
\hline 3 & 600 & -107 & 126.0747 & 168.115 \\
\hline 4 & 800 & -113 & 130.4757 & 174.115 \\
\hline 5 & 1000 & -97 & 133.8893 & 158.115 \\
\hline 6 & 1200 & -105 & 136.6785 & 168.115 \\
\hline 7 & 1400 & -107 & 139.0367 & 176.115 \\
\hline 8 & 1600 & -115 & 141.0794 & 172.115 \\
\hline 9 & 1800 & -111 & 142.8813 & 168.115 \\
\hline 10 & 2000 & -107 & 144.4931 & 164.115 \\
\hline 11 & 2200 & -103 & 145.9511 & 174.115 \\
\hline 12 & 2400 & -99 & 147.2822 & 168.115 \\
\hline 13 & 2600 & -113 & 148.5067 & 168.115 \\
\hline 14 & 2800 & -107 & 149.6404 & 164.115 \\
\hline 15 & 3000 & -107 & 150.6959 & 164.115 \\
\hline 16 & 3200 & -103 & 151.6832 & \\
\hline 17 & 3400 & -103 & 152.6106 & \\
\hline
\end{tabular}

\subsection{Discussion}

The experimental and simulated values for the various cases are close. It is observed that the simulated values which was obtained using Hata- Okumura model is much closer to the experimental values at a higher frequency. This validates the Hata-Okumura model. Effectively, this result shows that Hata-Okumura model is much accurate during the frequency range of $1920 \mathrm{MHz}-1955 \mathrm{MHz}$. Also, the tables show that path loss under the transmission 
line is higher compared to areas not covered by pylons. This is due to the fact that several communities in Lagos and other parts of Nigeria are criss-crossed by Pylon (transmission line). Network services in this community are very poor as a result of this pylon. It is therefore recommended that service providers should use higher power for Base Station located in such vicinity.

\section{CONCLUSION}

The studies compared simulated and experimental result for path loss in areas where there are no pylons with areas where there are pylons. This studies shows that areas with pylons have greater path loss compared with areas with no pylons. It is therefore recommended that power levels of base station operating in this area should be increased. This can be done using antenna with higher gain. Conclusively, this study show that there is a need to consider the atmospheric factor in propagation prediction models in order to achieve effective planning of cellular networks of next generation communication systems.

\section{REFERENCES}

[1] Worgu, S., Comparative evaluation of the path loss prediction performance Hata-Okumura path loss model for urban, suburban and rural areas, International Journal of Systems Science and Applied Mathematics, vol. 2, no.1, 2017, p. 42-50.

[2] Rappaport, T., Wireless communications principles and practice, Second edition, Prentice Hall, ISBN: 0130422320.

[3] Phaiboon, S., Phokharatkul, P., Path loss prediction for low-rise buildings with image classification on 2-d aerial photographs, Progress in Electromagnetics Research, PIER 95, 2009, p. 135-152.

[4] Nagendra, S., Thakur, T., Effects of clutters on path loss proposed by Walfisch-Ikegami propagation model, Proceedings of the Eleventh National Conference on Communications, NCC, 2005, p. 617-620, 2005.

[5] Emmanuel, C. A., Ezeh, N. G., Osuji, U., Cellular Mobile Signal Propagation; Effects of EIRP and Antenna Gain, Journal of Emerging Trends in Computing and Information Sciences, vol. 5, no. 3, 2014, p. $172-177$.

[6] Pooja, N., Anitha, R., Hanchinal, C. S., Optimized Path loss model for the effects of Environmental Factors on Mobile Signal Strength, International Research Journal of Engineering and Technology (IRJET), vol. 5, no. 4, 2008 .

[7] Sharma, H. K., Sahu, S., Sharma, S., Enhanced Cost231 W.I. Propagation Model in Wireless Network, International Journal of Computer Applications, vol. 19, no. 6, 2011.

[8] Casaravilla, J., Dutra, G., Pignataro, N., Acuna, J., Propagation Model for Small Macro cells in Urban Areas, IEEE Transactions on Vehicular Technology, vol. 58, no. 7, 2009. 